\title{
Robustness of a meta-network to alternative habitat loss scenarios
}

\section{Santos, Micaela}

2021-01

Santos , M , Cagnolo , L , Roslin , T , Ruperto , E , Bernaschini , M L \& Vázquez, D 2021 , '

Robustness of a meta-network to alternative habitat loss scenarios ', Oikos, vol. 130 , no. 1 , pp. 133-142 . https://doi.org/10.1111/oik.07835

http://hdl.handle.net/10138/334902

https://doi.org/10.1111/oik.07835

acceptedVersion

Downloaded from Helda, University of Helsinki institutional repository.

This is an electronic reprint of the original article.

This reprint may differ from the original in pagination and typographic detail.

Please cite the original version. 


\section{Robustness of a meta-network to alternative habitat loss scenarios}

Micaela Santos ${ }^{1}$, Luciano Cagnolo ${ }^{2}$, Tomas Roslin ${ }^{3,4}$, Emmanuel F. Ruperto ${ }^{1}$, María Laura Bernaschini ${ }^{2}$, Diego P. Vázquez ${ }^{1,5}$

${ }^{1}$ Argentine Institute for Dryland Research, CONICET, Av. Ruiz Leal s/n, 5500 Mendoza, Argentina

${ }^{2}$ Institute for Multidisciplinary Plant Biology (IMBIV), Faculty of Exact, Physical and Natural Sciences, National University of Cordoba \& CONICET, Av. Vélez Sarsfield 1611, 5000 Córdoba, Argentina

${ }^{3}$ Department of Ecology, P.O. Box 7044, Swedish University of Agricultural Sciences SE-750 07 Uppsala, Sweden

${ }^{4}$ Department of Agricultural Sciences, PO Box 27 (Latokartanonkaari 5), FI-00014 University of Helsinki, Finland

${ }^{5}$ Faculty of Exact and Natural Sciences, National University of Cuyo, Padre Jorge Contreras 1300, M5502JMA Mendoza, Argentina

Corresponding author: Micaela Santos, Argentine Institute for Dryland Research, CONICET, Av. Ruiz Leal s/n, 5500 Mendoza, Argentina. E-mail: santos.micaela.ms@gmail.com

Decision date: $27-$ Sep-2020

This article has been accepted for publication and undergone full peer review but has not been through the copyediting, typesetting, pagination and proofreading process, which may lead to differences between this version and the Version of Record. Please cite this article as doi: [10.1111/oik.07835]. 


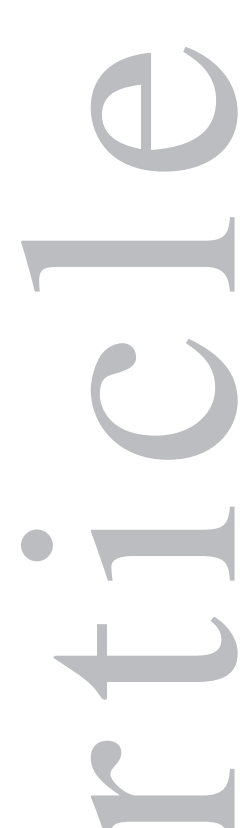

\section{Abstract}

Studying how habitat loss affects the tolerance of ecological networks to species extinction (i.e., their robustness) is key for our understanding of the influence of human activities on natural ecosystems. With networks typically occurring as local interaction networks interconnected in space (a meta-network), we may ask how the loss of specific habitat fragments affects the overall robustness of the meta-network. To address this question, for an empirical meta-network of plants, herbivores and natural enemies we simulated the removal of habitat fragments in increasing and decreasing order of area, age and connectivity for plant extinction and the secondary extinction of herbivores, natural enemies and their interactions. Meta-network robustness was characterized as the area under the curve of remnant species or interactions at the end of a fragment removal sequence. To pinpoint the effects of fragment area, age and connectivity, respectively, we compared the observed robustness for each removal scenario against that of a random sequence. The meta-network was more robust to the loss of old (i.e. long-fragmented), large, connected fragments than of young (i.e. recently fragmented), small, isolated fragments. Thus, young, small, isolated fragments may be particularly important to the conservation of species and interactions, while contrary to our expectations larger, more connected fragments contribute little to meta-network robustness. Our findings highlight the importance of young, small, isolated fragments as sources of species and interactions unique to the regional level. These effects may largely result from an unpaid extinction debt, in which case these fragments are likely to lose species over time. Yet, there may also be more long-lasting effects from cultivated lands (e.g. water, fertilizers, and restricted cattle grazing) and network complexity in small, isolated fragments. Such fragments may sustain important biological diversity in fragmented landscapes, but maintaining their conservation value may depend on adequate restoration strategies.

\section{Key Words}

Fragmented landscape, meta-network, plant-herbivore-natural enemy network, robustness 


\section{Introduction}

Habitat destruction is currently one of the major drivers of biodiversity loss at a global scale, because it leads to species extinction and often triggers co-extinction cascades (Barnes et al. 2017). Where species interact forming complex interaction networks (Gravel et al. 2019), both the structure and functioning of ecological networks are altered as a consequence of habitat loss and fragmentation (Grass et al. 2018). This may result in simpler, increasingly homogeneous landscapes with a progressive degradation of ecosystem services (Tscharntke et al. 2005). Yet, how species are lost from complex ecological networks embedded in fragmented landscapes is so far poorly known (Evans et al, 2013). Therefore, understanding how landscape transformation drives the extinction of species and their interactions is key for a sustainable use of natural ecosystems and to guarantee essential goods and services for human societies (Isbell et al. 2017).

With species tied into interaction networks, the local extinction of a population may lead to secondary extinctions of other populations that depend on it (Montoya et al. 2006). Network robustness refers to the tolerance of ecological networks to species extinctions (Dunne et al. 2002), so that a network will be more robust when it experiences fewer secondary extinctions after losing a particular species (Dunne et al. 2002; Memmott et al. 2004). In natural ecosystems, the order of species extinction is unlikely to be random, but rather to reflect species' differential sensitivity to perturbations (Larsen et al. 2005). Previous studies have shown that both mutualistic and antagonistic interaction networks are more robust to the extinction of randomly selected species than of highly connected species (i.e. generalists), as the extinction of 
generalists can lead to the secondary extinction of associated specialist species (Dunne et al. 2002; Memmott et al. 2004). Fortunately, the extinction of generalists is less likely in real, natural systems than in randomly assembled communities, since in nature well connected species tend to have a lower extinction risk because of the greater availability of alternative potential interaction partners than less connected species (specialists) (Biesmeijer et al. 2005). Yet, when the extinction of generalists does occur, which is likely under some realistic extinction scenarios, it can lead to many secondary extinctions (Srinivasan et al. 2007). Such more realistic sequences have been based on considering e.g. the nested distribution of species across habitats, where species-poor habitats are characterized by a subset of the species present in richer habitats (Srinivasan et al. 2007).

In sum, both fragment features and species sensitivity to habitat transformations may affect meta-community persistence in fragmented habitats. Multilayer networks represent a novel approach to study spatially fragmented communities and their inherent interdependence (Pilosof et al. 2017). Meta-networks are considered a multilayer representation of a set of ecological networks which are spatially distributed, connected by dispersal and influenced by colonization-extinction dynamics, and which in other words form a "network of networks" in a meta-community context (Hagen et al. 2012). Within a meta-community, highly connected local communities may sustain more species and larger populations than isolated communities; high connectivity, in turn, decreases the local extinction risk of species (MacArthur and Wilson 1967), especially of species at high trophic levels (Holt et al. 1999; Melián and Bascompte 2002). However, species persistence in landscapes depends largely on resource availability within their dispersal scale, which is related to fragment connectivity (e.g., distance 
among fragments) and the amount of habitat available (e.g., area of neighboring fragments) (Hanski 1999). Furthermore, species extinction as a consequence of habitat loss and fragmentation may not be immediate, especially for species with long generation times such as perennial plants (Figueiredo et al. 2019). Therefore, fragment age, i.e. the time elapsed since fragmentation, may be a key factor of current species composition in the landscape beyond connectivity and fragment size (Aguilar et al. 2018) due to a possible extinction debt still being unpaid (Kuussaari et al. 2009).

The effect of habitat loss on the robustness of ecological networks has been previously studied by simulating the loss of habitat patches of identical characteristics by simulating species extinctions in habitat patches in both simulated (Fortuna and Bascompte 2006) and real landscapes (Guardiola et al, 2018; Grass et al, 2018). Nonetheless, how the complete disappearance of specific habitat fragments in a real landscape affects meta-network robustness, is still poorly understood (Evans et al. 2013). Although we know that the loss of heterogeneous habitats may affect metanetwork robustness (Evans et al. 2013), the effect of the loss of fragments with different spatio-temporal attributes surrounded by an anthropogenic matrix, such as fragment connectivity and age, adds potentially important ramifications and it has not been studied before. Furthermore, considering matrix composition and configuration explicitly may provide more information on meta-network robustness, especially in heterogonous landscapes (e.g., with several kinds of crops or a diverse topography).

Our aim is to assess meta-network robustness to the removal of fragments with different areas, ages and connectivities, and whether such robustness varies among trophic groups and interaction types. Because our study area has a highly homogenous matrix 
composition (mostly vineyards), we assumed connectivity was affected equally by the habitat matrix through the landscape. We hypothesized that if the contribution of fragments to meta-network robustness depends on their area, age and connectivity within habitat network, then the removal order of fragments according to these attributes should translate into meta-network robustness. We expected that the early loss of highly connected, large, young (i.e. recently isolated) fragments leads to a greater decrease in meta-network robustness than the loss of more isolated, smaller, older fragments. Furthermore, we expected that decreases in meta-network robustness will have more extreme values when considering species with increasing dependence to their resources (determined by trophic level or trophic specialization) and habitat specialization. Our study aims at shedding light on the relative impact of specific fragments in a meta-network context, and may thereby allow the identification of key versus redundant fragments (high or low contribution to robustness, respectively) information essential to habitat management and biological conservation of multiple species (Urban et al. 2009).

\section{Methodology}

\section{Study area}

To assess the relative contribution of fragments to meta-network robustness, we identified habitat fragments within an area of a fragmented dryland habitat in Valle de Uco, Mendoza Province, central-western Argentina. This region is characterized by an intense habitat modification since ca. 20 years ago, which has resulted in strong fragmentation, degradation and loss of the native Monte Desert ecosystem which natural vegetation is predominantly xerophytic. Remnant fragments are mostly 
surrounded by vineyards (personal observation) and to a lesser extent by other types of agriculture (e.g., walnut orchards) or urbanized cover (e.g., roads or human settlements). In this setting, we defined a habitat fragment as any patch of remnant native vegetation with more than $70 \%$ of its perimeter surrounded by strongly modified habitat (e.g., agricultural land, roads, human settlements) and without indication of strong human disturbance (e.g., vegetation clearing). We identified 19 natural fragments within the target region of ca. 50,000 hectares, and used QGIS 2.18 free software to measure fragment area and geographical distance among all fragments (the shortest edge-to-edge distance between fragments). We did not detect spatial autocorrelation in fragment area (Moran's I $=-0.06, \mathrm{p}$-value $=0.83$ ). Fourteen fragments were sampled with permission from land owners, while the other five sites were left unsampled because of logistical constraints. These unsampled fragments and the neighboring continuous vegetation were included in all estimates of fragment connectivity for a full characterization of the landscape, so as to consider all possible dispersal routes among fragments. To assess when habitat fragmentation had taken place, we used available historical maps of the study area in GoogleEarth 7.3 (from 2004 to present) to determine fragment age (Supplementary material Appendix 1, Table A4 and Table A5). Furthermore, we explored correlations for these three fragment characteristics (Supplementary material Appendix 1, Fig. A3).

\section{Sampling and meta-network construction}

To build the meta-network we considered all plant-herbivore-natural enemy interactions (as presence-absence data) recorded in 14 habitat fragments (Supplementary material Appendix 1, Fig. A4), which involved plant species (herbs and shrubs), endophagous herbivores (gall makers or gall inquilines, and leaf miners), ectophagous herbivores 
(scale insects and aphids), and their natural enemies (parasitoids, hyperparasitoids and predators). We sampled plant-herbivore interactions at the centroid of each fragment along two $50 \mathrm{~m} \times 2 \mathrm{~m}$ transect bands during the spring and summer of two consecutive years (2015-2016 and 2016-2017). We kept the sampling area constant to avoid confounding the true effects of fragment area with sampling effort. We kept the immature and/or parasitized herbivores in the lab (galls, mines, parasitized aphids and scale insects) until herbivore and parasitoid adult emergence, which allowed recording herbivore-parasitoid interactions. We identified all species and morphospecies emerged from galls, mines and parasitized aphids and scale insects, then we assigned them to the most likely trophic guild; alternatively, we identified galls and mines through external morphology when adult herbivores did not emerge in the lab. Furthermore, as insects associated to galls were highly diverse, it was not possible to determine whether a given species was a gall maker, a predator or parasitoid of the gall maker, or an opportunistic occupier of the gall, therefore we decided to consider parasitoids and predators interacting with the whole gall (Supplementary material Appendix 1, Table A1; for details see Santos et al. 2019).

\section{Measuring of meta-network robustness to alternative scenarios of fragment removal}

To explore fragment contribution to meta-network robustness, we sorted the 14 habitat fragments according to their area (A), age (E) and six connectivity metrics combining geographical distances among fragments (D), neighboring area (A) and similarity in plant resources among fragments (S) (see correlations in Supplementary material Appendix 1, Fig A3). We described fragment connectivity $\left(\mathrm{C}_{\mathrm{i}}\right)$ in its full version as $C_{i}=\sum e^{D_{i j}} A_{j} S_{i j}$ (equation 1), 
where $j$ represents a neighboring fragment, and we used D, DA, DAS, DS, SA and S as components of the six alternative connectivity metrics (more details in Santos et al. 2019). We simulated fragment removal in increasing order-from small to large, young to old, little to highly connected fragments — and decreasing — from large to small, old to young, highly to little connected fragments (Fig. 1c).

For each fragment removal, we considered secondary extinctions of herbivores and tertiary extinctions of natural enemies as consequence of the local extinction of plant resource, and calculated remnant species richness and trophic interactions. Therefore, we simulated extinctions for unique species and interactions occurring in each fragment, while redundant interactions (present in other fragments) remained in the meta-network; this simulation implies that we assessed the static structure of the meta-network after a given amount of time, during which the metacommunity was influenced by landscape structure through colonization and extinction events. We assumed that interactions exclusively recorded at a given site do not occur in other habitat fragments even if the plant resources or interacting partners were present at those sites. This assumption is based on the idea that although an interaction is biologically plausible, it might not occur in all fragments as interactions have a stochastic component (Gravel et al. 2019); however, we acknowledge that the possibility of false negatives created by low temporal sampling (2 consecutive years). Thus, if for example a parasitoid had been recorded parasitizing a specific herbivore species only in one fragment, and this fragment was then removed, then that interaction disappears from the meta-network. This mechanism would occur even if the same herbivore and parasitoid species were present in other fragments interacting with other partners; thus for this example, the interaction is lost but the species is not. Alternatively, even if a resource was present in 
a fragment but its consumer was not recorded there, it was assumed that no interaction occurred; this is based on the idea that pairs of consumer-resource species could cooccur in some fragments but not in others due to dispersal limitations, i.e. we assumed differential dispersal among species.

To compare habitat loss across species groups and interaction types, we calculated minimum and maximum average of extinctions for the best and the worst scenarios, respectively, considering the removal of a half of the fragments. Then, we calculated meta-network robustness as the area under the curve of remnant species and interactions for a specific removal sequence (Memmott et al. 2004) for each trophic guild and interaction type. To order fragments by age sequence we randomly selected fragments of increasing or decreasing ages; when two or more fragments had the same ages, we sampled them with equal probability (100 samples), and we kept the increasing and decreasing sequences with the highest and lowest robustness, respectively. Finally, we compared observed robustness for each removal rule against calculated robustness for 100 random fragment removals (code provided in Supplementary material Appendix 2). We interpreted the relative contribution of fragments with certain attributes as determinants of meta-network robustness. In addition, we calculated connectance and linkage density in local networks using the bipartite package of $\mathrm{R}$ (Dormann et al. 2009) as a complementary description of network complexity in fragments which reflect changes in the frequency of different interactions. Connectance is a qualitative network descriptor historically associated to network complexity in the literature (May, 1972), but it is sensitive to sampling effort, while quantitative descriptors have the potential to overcome this problem (Bersier et al. 2002); these metrics describe the extent to which species are connected: connectance as a ratio of the number of species (Dunne et al. 
2002) and linkage density as the marginal totals-weighted diversity of interactions per species (Bersier et al. 2002). All analysis were carried out using R 3.5.1 (R Core Team, 2019).

\section{Results}

\section{Meta-network composition}

The meta-network comprised 14 habitat fragments, 40 plants species harboring 40 gall types, 26 mine types, 30 morphospecies of scale insects, 22 morphospecies of aphids (all of these based on external morphology), and 166 parasitoid species or morphospecies based on taxonomic descriptions. Furthermore, we identified 78 herbivore species among adults emerged from mines and galls, including "inquilines" i.e. insects which consume gall tissue (Askew 1980) and we recorded other herbivores probably "tourists" which may have been associated incidentally (Supplementary material Appendix, Table A1 and Table A2). We found some congeneric plants without associated insects that could be a result of insufficient sampling effort to detect interactions related to low plant abundances or the specificity of herbivores to plant species level (not genera), which is quite common in endophage herbivores. We usually found herbivores on plants from medium to high abundance, mainly woody plants native to the Monte Desert (see Supplementary material Appendix 1, Table A2). Although low plant abundances could have prevented the detection of some interactions, given that most interactions were found on abundant plants, we believe that this detection problem should not alter the results.

\section{Relative contribution of habitat fragments to meta-network robustness}


Removal of a half of the fragments in all habitat loss scenarios resulted in the extinction of between $16 \%$ and $38.6 \%$ of species in average (Fig. 1a). Removing fragments in order of decreasing age lead to the lowest extinction rates (highest robustness), with plants experiencing the fewest extinctions $(\sim 10 \%)$ and parasitoids the highest $(\sim 20 \%)$ (Supplementary material Appendix, Fig. A1ac). At the same time, removing fragments in order of increasing $S$ connectivity lead the highest rate of species extinctions, with plants suffering the fewest proportional extinctions $(\sim 32 \%)$ and predators and herbivores the highest ( 45\% and $42 \%$, respectively) (Supplementary material Appendix, Fig. A1y). Furthermore, we found between $27.1 \%$ and $48.3 \%$ of interaction extinctions on average when we removed half of the fragments (Fig. 1b). The fewest extinctions were recorded when fragments were removed in order of decreasing age, with plant-herbivore interactions being the least affected $(\sim 18 \%)$ and herbivoreparasitoid interactions the most affected ( 34\%) (Supplementary material Appendix, Fig. A1ae). In contrast, the greatest impact was observed when fragments were removed in order of increasing $D$ connectivity (see equation 1), with herbivore-predator interactions having the highest rate of extinctions $(\sim 58 \%)$, followed by herbivoreparasitoid interactions ( \% \%8) and plant-herbivore interactions $(\sim 38 \%)$ (Supplementary material Appendix, Fig. A1g). We have found the same trend when we split the data set by years (Supplementary Material Appendix 1, Fig. A6), i.e. the meta-network was more robust to the loss of old, large, connected fragments than of young, small, isolated fragments.

Considering a meta-network composed by all species groups and trophic interaction types as a whole, we found higher robustness for scenarios where habitats were lost in a decreasing order of age and connectivity including some components (Table 1) than for 
scenarios of random fragment removal. In other words, we observed a relative increase in robustness for the cases where we first eliminated the largest, oldest or most connected fragments (with connectivity characterized by any connectivity metric; Fig. 2 red triangles). In contrast, we found lower robustness than expected for random fragment removal when we first eliminated small, young and isolated fragments (Fig. 2 blue triangles), i.e. for scenarios of habitat loss in an increasing order (Table 1). Supporting higher relative robustness for decreasing than increasing age scenario, we observed less plant richness in old fragments, considering both woody native plants and herbs, thus when these fragments were removed first the meta-network did not experience too much secondary and tertiary extinctions (Supplementary material Appendix, Fig A2).

The best and worst scenarios of habitat loss differed among species groups and trophic interaction types (Table 1). Nonetheless, they all shared an increasing trend of metanetwork robustness under a decreasing order of fragment removal and a decreasing trend under an increasing order of fragment removal according to fragment features. Furthermore, meta-network robustness decreased with trophic level for species, and even more strongly for interactions. For species, young, dissimilar fragments in plant composition contributed more to the maintenance of plants, herbivores (except for aphids) and predators, while geographically isolated fragments contributed more for parasitoids. For interactions, dissimilar fragments in plant composition contributed more in preventing extinctions of plant-herbivore interactions, while geographical connectivity and area of neighboring fragments contributed more to maintaining interactions between herbivores and natural enemies. In addition, we found more complex plant-herbivore networks (greater linkage density) in isolated fragments and 
more complex herbivore-parasitoid networks in small fragments (Supplementary material Appendix 1, Table A3).

\section{Discussion}

We assessed the relative contribution of habitat fragment with different area, connectivity and age to meta-network robustness facing alternative realistic scenarios of habitat loss. We found that early removal of young, small, isolated fragments resulted in a higher number of plant primary extinctions, secondary extinctions of herbivores and tertiary extinctions of their natural enemies, as well as the extinction of trophic interactions. Contrary to expectation, neither fragment area nor connectivity contributed to meta-network robustness. Thus, the key role of young, small, isolated fragments for meta-network robustness highlights their value as sources of unique species and interactions at the regional level, which, if correctly managed, may contribute to biological conservation and community restoration in fragmented landscapes. Although establishing dispersal limitations of multiple species in a metacommunity may be unfeasible, we are aware that including spatial dynamics in future studies would add realism to predictions. However, our approach is the first step to understand the metanetwork structure and their consequences on the robustness for multiple species and their trophic interactions.

\section{Contribution to meta-network robustness of young, small and isolated fragments}

The robustness of fragment removal in sequences from small to large, young to old and little to highly connected was considerably lower than the mean robustness of random fragment removal, and in most cases lay outside of the confidence limits of outcomes 
expected under the random scenario. These results suggest that species and interactions in small, young, little connected fragments are not redundant and that therefore, their elimination should lead to the extinction of unique species and interactions at the regional level. Consistent with our predictions, we found that plant and animal composition in young fragments, as a result of short periods of disturbance, might contribute with more unique assemblages of species and interactions than old fragments (Valiente-Banuet et al. 2015), which suggests a possible unpaid extinction debt. In fact, meta-network robustness increased substantially when old fragments were removed first, which harbor lower plant richness, including both woody, native, long-lived plants and non-woody, ruderal, short-lived plants (herbs) (Supplementary material Appendix 1, Fig. A2). However, and contrary to our expectations, small, isolated fragments contributed more than large, well connected fragments, which might be related to the specificities of our study area or network structure.

In addition to a potential extinction debt, several non-mutually exclusive factors may add to the high contribution to meta-network robustness by the smallest, most isolated fragments. The Monte ecoregion is characterized by low precipitation, extreme temperatures and xerophytic vegetation, while cultivated lands under irrigation represent "oases" within the desert; thus, it is likely that small- or medium-sized fragments surrounded by crops benefit from water and fertilizer supplies. In this context, the species-energy relationship may be explaining the contribution of small fragments to meta-network robustness better than the species-area relationship, because it replaces area by an estimate of available energy, such as productivity, to predict species richness (Wright 1983). Therefore, the benefit obtained through energy from water and fertilizers entering a fragment could result in higher plant richness (e.g. herb 
proliferation) through increased number of individuals ("more individuals hypothesis"; Srivastava and Lawton 1998) or increased plant growth and thus more resources available to herbivores. In support to this theory, we found slightly higher richness of ruderal herbs in smaller fragments but not of woody plants (Supplementary material Appendix 1, Fig A2); we also detected changes in the plant composition of the fragments considering all species plants or only herbs (Supplementary material Appendix 1, Fig A5), although we did not find high correlation with fragment area. However, richness of plant-herbivore interactions decreased with area. These results suggest that supplies from cultivated land could be contributing moderately to the proliferation of different herbs on small fragments, but not of herbivores, as a result of transient dynamics (Ovaskainen \& Hanski, 2002), and thus it may affect meta-network robustness on some species of the basal trophic level but it does not on interactions and species of higher trophic levels.

Furthermore, there seems to be a relationship between fragment size and isolation and the type and intensity of disturbances to which they are exposed. In fact, based on field observations, we found that habitat fragments completely surrounded by crops usually prevent access by domestic cattle, unlike fragments limited by roads or urban areas, which are frequently larger and better geographically connected to continuous habitat. This restriction in more isolated fragments could represent a benefit to native vegetation and associated insect community because it avoids negative effects of cattle on natural vegetation (Chillo and Ojeda 2014). Therefore, small, isolated fragments, which are apparently less perturbed by cattle, can result in richer plant communities with larger populations and thus, a greater contribution to meta-network robustness. Considering connectivity based only on geographical distances, we found no evidence of this 
mechanism (Supplementary material Appendix 1, Fig. A2). Future studies should assess whether more realistic metrics of connectivity including matrix characteristics and species mobility lead to different conclusions.

An alternative explanation concerns differences in network complexity among fragments. Robustness is a component of network stability, and while a body of evidence supports the notion that network complexity hampers stability through effects of direct and indirect interactions (Fox and Olsen 2000; Melián and Bascompte 2002; Thébault and Fontaine 2010; Vieira and Almeida-Neto 2015; McWilliams et al. 2019), there is also evidence supporting the opposite idea (Dunne et al. 2002; Allesina and Tang 2012); we found more complex networks (higher connectance and linkage density) in small, isolated fragments, which had a higher contribution in meta-network robustness (Supplementary material Appendix 1, Table A3). Thus, our results agree with the notion that greater complexity enhances stability (robustness in this case) and reinforces the assumptions about lower perturbation intensity in those habitat fragments. An alternative explanation is related to changes in network structure through behavioral responses such as rewiring (switching of interaction partners), which can occur as an adaptive strategy against habitat modifications (Tylianakis and Morris 2017). Simulation studies have shown that interaction rewiring enhances robustness in mutualistic networks (Ramos-Jiliberto et al. 2012). Therefore, as rewiring can be expected to be particularly prevalent in more modified habitats (small, isolated), where "whimsical" species combinations lead to new interactions, those fragments may contribute to enhance meta-network robustness.

\section{Meta-network robustness to different species and trophic interactions}


We showed that habitat loss affects meta-network robustness for species belonging to different guilds and their trophic interactions. Plants were more robust than animals in almost all the scenarios evaluated; this is hardly surprising, since plants represent the basal trophic level on which the other trophic levels depend. Thus, higher trophic levels generally show higher susceptibility to habitat loss (Holt et al. 1999, Roslin et al. 2014). However, we showed that "best" (i.e. least devastating) scenarios of habitat loss for herbivores and natural enemies were different and we did not detect large differences between their robustness; these results suggest that both trophic levels may have similar tolerance to habitat loss and they relate with landscape in a different way.

Parasitoids showed greater robustness than herbivores, especially in scenarios that involved fragment removal of decreasing connectivity by geographical distances (weighted or not by area and similarity in plant composition). This result may be due to parasitoids having a wider regional distribution (higher mobility) and greater trophic generalization than herbivores (Holt et al. 1999). If so, then elimination of more connected fragments from the landscape may not reflect into any higher rates of secondary extinctions among parasitoid, simply because they are widely distributed among fragments (Holt et al. 1999). As an alternative, by being generalists the parasitoids may persist by developing in alternative herbivore host species, while specialist herbivores become extinct when their plant resources disappear (Cagnolo et al. 2009). These explanations also agree with the results that we found for trophic interactions in habitat loss scenarios based on connectivity by distances; the greater extinction rates (i.e. lower robustness) observed for herbivore-parasitoid interactions than plant-herbivore interactions suggest that, although parasitoids suffered few species 
extinctions, they effectively lost more interactions, so each loss of a parasitoid species implied many interactions lost.

\section{Conclusions}

The contribution of small, young, isolated fragments to meta-network robustness highlights these habitats as sources of unique species and interactions at the regional level. Therefore, these fragments could contribute to biodiversity conservation and the restoration of fragmented landscapes. However, the apparent occurrence of an extinction debt in young fragments suggests that the value of these fragments could change over time. Thus, the spatio-temporal heterogeneity of fragment contribution to meta-network robustness provides a unique opportunity for restoration in old fragments through the incorporation of biological corridors increasing landscape connectivity, while small, isolated fragments may represent a source of species to more degraded fragments.

\section{Acknowledgement}

We thank to Hugo J. Marrero for help the field and Juan José Martínez for help in taxonomic identifications. We also thank to Melanie Roy for an stimulating talk that helped to enrich discussion section of this work.

\section{Funding}

This work was supported by FONCYT, Argentina (PICT-2014-3168). MS was additionally supported by a doctoral fellowship from CONICET, Argentina. 


\section{Author contributions}

MS and EFR collected field data. MLB and LC helped in taxonomic identifications.

MS, LC and DPV conceived and designed the study. MS and DV wrote the code and carried out the statistical analyses. All authors helped draft the manuscript and gave final approval for publication.

\section{Conflicts of interest}

No author has any conflict of interest.

\section{References}

Aguilar, R. et al. 2018. Unprecedented plant species loss after a decade in fragmented subtropical chaco serrano forests. - PLoS One, 13, e0206738.

Allesina, S. and Tang, S. 2012. Stability criteria for complex ecosystems. - Nature, 483: 205-208.

Askew, R.R. 1980. The diversity of insect communities in leafmines and plant galls. - J. Anim. Ecol., 49: $817-829$.

Barnes, A.D. et al. 2017. Direct and cascading impacts of tropical land-use change on multi-trophic biodiversity. - Nat. Ecol. Evol., 1: 1511-1519.

Bersier, L. F. et al (2002). Quantitative descriptors of food-web matrices. - Ecology, 83: 2394-2407

Biesmeijer, J.C. et al. 2005. Parallel Declines in Pollinators and Insect-Pollinated Plants in Britain and the Netherlands. - Science, 309: 570-574.

Cagnolo, L. et al. 2009. Habitat fragmentation and species loss across three interacting trophic levels: Effects of life-history and food-web traits. - Conserv. Biol., 5: 1167-1175.

Chillo, V. and Ojeda, R. 2014. Disentangling ecosystem responses to livestock grazing in drylands. Agriculture, Ecosyst. Environ., 197: 271-277.

'This article is protected by copyright. All rights reserved.' 
Dormann, C.F. et al. 2009. Indices, graphs and null models: Analyzing bipartite ecological networks. -

Open Ecol. J., 2: 7-24.

Dunne, J.A. et al. 2002. Network structure and biodiversity loss in food webs: robustness increase with connectance. - Ecol. Lett., 5: 558-567.

Evans, D.M. et al. 2013. The robustness of a network of ecological networks to habitat loss. - Ecol. Lett., 16: $844-852$.

Figueiredo, L. et al. 2019. Understanding extinction debts: spatio-temporal scales, mechanisms and a roadmap for future research. - Ecography, 42: 1-18.

Fortuna, M. and Bascompte, J. 2006. Habitat loss and the structure of plant-animal mutualistic networks. - Ecol. Lett., 9: 281-286.

Fox, J.W. and Olsen, E. 2000. Food web structure and the strength of transient indirect effects. - Oikos, 90: 219-226.

Grass, I. et al. 2018. Past and potential future effects of habitat fragmentation on structure and stability of plant-pollinator and host-parasitoid networks. - Nat. Ecol. Evol., 2: 1408-1417.

Gravel, D. et al. 2019. Bringing Elton and Grinnell together: a quantitative framework to represent the biogeography of ecological interaction networks. - Ecography, 42: 401-415.

Guardiola, M. et al. 2018. Do asynchronies in extinction debt affect the structure of trophic networks? A case study of antagonistic butterfly larvae-plant networks. - Oikos, 127: 803-813.

Hagen, M. et al. 2012. Biodiversity, Species Interactions and Ecological Networks in a Fragmented World. - Adv. Ecol. Res. 1st edn. Elsevier Ltd, 46:89-120.

Hanski, I. 1999. Habitat Connectivity, Habitat Continuity, and Metapopulations in Dynamic Landscapes. - Oikos, 87: 209-219.

Holt, R.D. et al. 1999. Trophic Rank and the Species-Area Relationship. - Ecology, 80: 1495-1504.

Isbell, F. et al. 2017. Linking the influence and dependence of people on biodiversity across scales. - Nature, 546: 65-72.

'This article is protected by copyright. All rights reserved.' 
Kuussaari, M. et al. 2009. Extinction debt: a challenge for biodiversity conservation. - Trends Ecol. Evol., 24: 564-571.

Larsen, T.H. et al. 2005. Extinction order and altered community structure rapidly disrupt ecosystem functioning. - Ecol. Lett., 8: 538-547.

MacArthur, R.H. and Wilson, E.O. 1967. The theory of island biogeography. - Press, Pri. Princeton.

May, R.M, 1972, Will a large complex system be stable? - Nature 238: 413-414.

McWilliams, C. et al. (2019). The stability of multitrophic communities under habitat loss. - Nat. Commun., 10: 1-11.

Melián, C.J. and Bascompte, J. 2002. Food web structure and habitat loss. - Ecol. Lett., 5: 37-46.

Memmott, J. et al. 2004. Tolerance of pollination networks to species extinctions. - Proc. Biol. Sci., 271: 2605-2611.

Montoya, J.M. et al. 2006. Ecological networks and their fragility. - Nature, 442: 259-264.

Ovaskainen, O. \& Hanski, I. 2002. Transient dynamics in metapopulation response to perturbation. Theor. Popul. Biol., 61: 285-295.

Pilosof, S. et al. 2017. The multilayer nature of ecological networks. - Nat. Ecol. Evol., 1, 0101.

“QGIS Development Team (2019). QGIS Geographic Information System. Open Source Geospatial Foundation Project

Ramos-Jiliberto, R. et al. 2012. Topological plasticity increases robustness of mutualistic networks. - J. Anim. Ecol., 81: 896-904.

Roslin, T. et al. 2014. Species-area relationships across four trophic levels - decreasing island size truncates food chains. - Ecography, 37: 443-453.

Santos, M. et al. 2019. Landscape connectivity explains interaction network patterns at multiple scales. Ecology, 100: 1-8.

Srinivasan, U. et al. 2007. Response of complex food webs to realistic extinction sequences. - Ecology, 88: 671-682.

'This article is protected by copyright. All rights reserved.' 
Srivastava, D.S. and Lawton, J.H. 1998. Why more productive sites have more species: An experimental test of theory using tree-hole communities. - Am. Nat., 152: 510-529.

Thébault, E. and Fontaine, C. 2010. Stability of ecological communities and the architecture of mutualistic and trophic networks. - Science, 329: 853-6.

Tscharntke, T. et al. 2005. Landscape perspectives on agricultural intensification and biodiversity - Ecosystem service management. - Ecol. Lett., 8: 857-874.

Tylianakis, J.M. and Morris, R.J. 2017. Ecological Networks Across Environmental Gradients. - Annu. Rev. Ecol. Evol. Syst, 48: 25-48.

Urban, D.L. et al. 2009. Graph models of habitat mosaics. - Ecol. Lett., 12: 260-273.

Valiente-Banuet, A. et al. 2015. Beyond species loss: The extinction of ecological interactions in a changing world. - Funct. Ecol., 29: 299-307.

Vieira, M.C. and Almeida-Neto, M. 2015. A simple stochastic model for complex coextinctions in mutualistic networks: Robustness decreases with connectance. - Ecol. Lett., 18: 144-152.

Wright, D.H. 1983. Species-Energy Theory: An Extension of Species-Area Theory. - Oikos, 41: 496506.

'This article is protected by copyright. All rights reserved.' 


\section{Figure Legends}

Fig. 1 Average percentage of a) species (plants, herbivores, parasitoids and predators) b) interaction extinctions (plant-herbivore, herbivore-parasitoid and herbivore-predator) under alternative scenarios of habitat loss in a fragmented Monte shrubland (Valle de Uco, Mendoza) illustrated in c). The bars show extinctions as a percentage of extinct species and interactions after half of the fragments had been removed in increasing or decreasing order according to fragment age (E), area (A), connectivity by geographical distances (D), connectivity by similarity in plant composition (S) and combinations of the last three attributes. Dotted lines show maximum and minimum percentage of species and interaction extinctions.
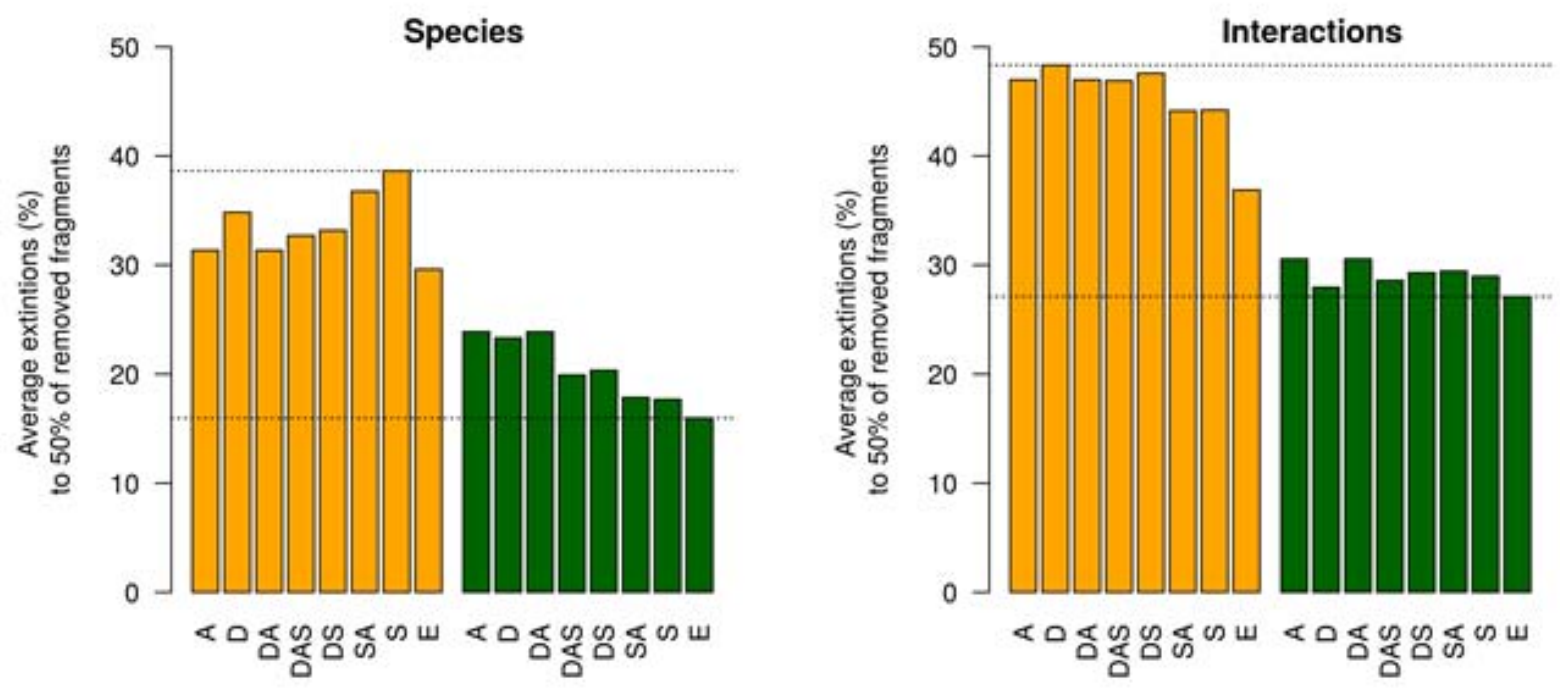

= Increasing order of removal

- Decreasing order of removal 


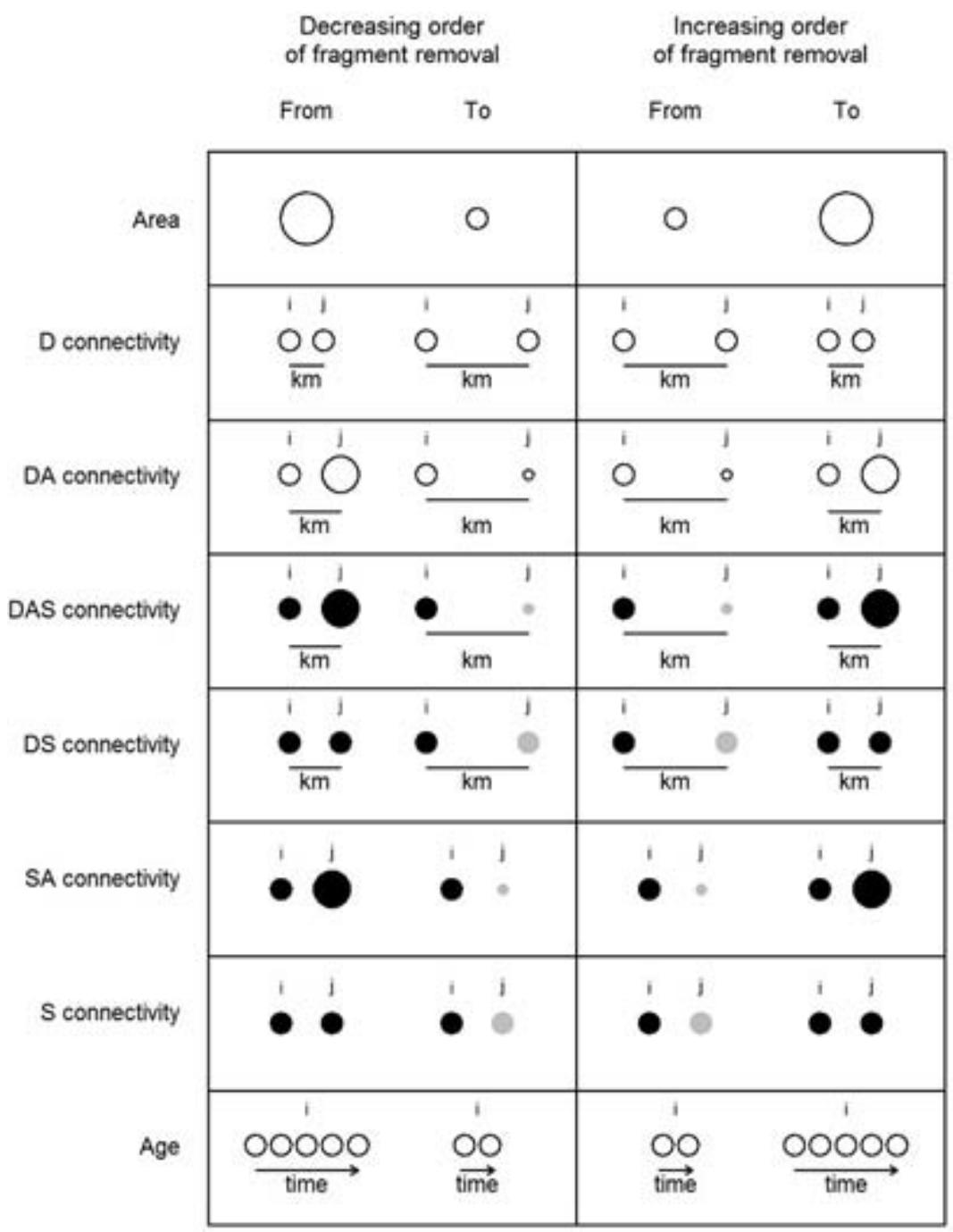

'This article is protected by copyright. All rights reserved.' 
Fig. 2 Meta-network robustness in a fragmented habitat of native Monte (Valle de Uco, Mendoza) for different species groups and trophic interactions under alternative habitat loss scenarios against random removal. Each panel shows the median with $95 \%$ confidence limits from the random scenario with fragments removed in increasing (blue triangles) and decreasing (red triangles) order according to (a) age, (b) area and (c-h) connectivity.

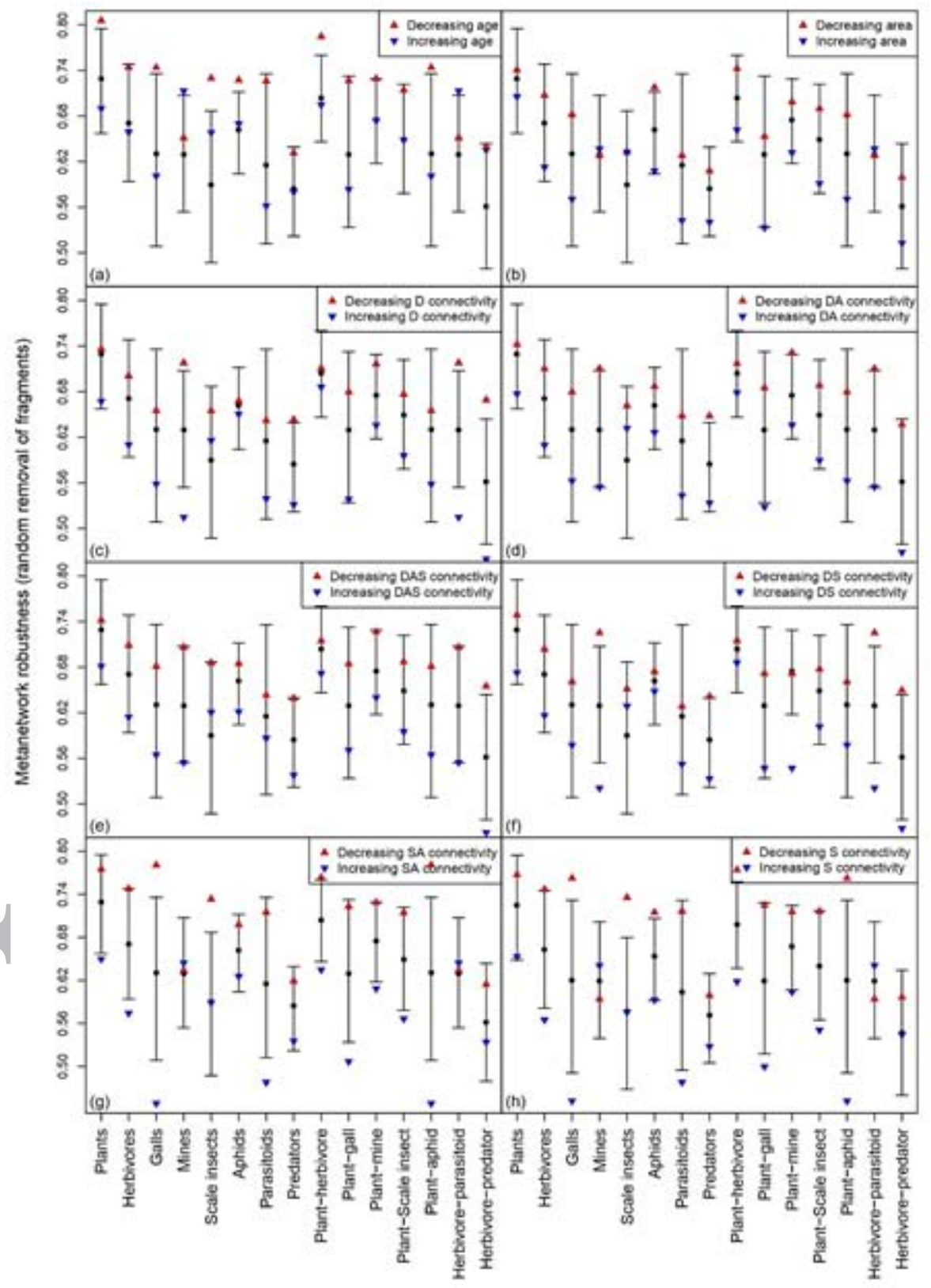

'This article is protected by copyright. All rights reserved.' 


\section{Table Legend}

Table 1 Summary table of the "best" and the "worst" scenarios of habitat loss according to age, area and connectivity (see references in Fig. 1) for increasing and decreasing removal order, considering the highest meta-network robustness $(\mathrm{R})$ as the best scenario and the lowest robustness as the worst.

\begin{tabular}{c|l|c|c|c|c|}
\multirow{2}{*}{ Meta-network } & \multicolumn{4}{|c|}{ Habitat loss scenarios } \\
\cline { 2 - 6 } Species & Best & R & Worst & R \\
\hline \multirow{5}{*}{} & Plants & $\mathrm{E}$ & 0.81 & $\mathrm{SA}$ & 0.65 \\
& Herbivores & $\mathrm{S}$ & 0.75 & $\mathrm{SA}$ & 0.57 \\
& Galls & $\mathrm{E}$ & 0.78 & $\mathrm{~S}$ & 0.63 \\
& Mines & $\mathrm{SA}$ & 0.78 & $\mathrm{SA}$ & 0.45 \\
& Scale insects & $\mathrm{S}$ & 0.73 & $\mathrm{SA}$ & 0.51 \\
& Aphids & $\mathrm{DS}$ & 0.73 & $\mathrm{D}$ & 0.51 \\
& Parasitoids & $\mathrm{DA}$ & 0.73 & $\mathrm{SA}$ & 0.61 \\
& Predators & $\mathrm{S}$ & 0.74 & $\mathrm{~S}$ & 0.59 \\
\hline \multirow{5}{*}{ Interactions } & $\mathrm{S}$ & 0.72 & $\mathrm{~S}$ & 0.57 \\
& Plant-herbivore & $\mathrm{E}$ & 0.73 & $\mathrm{~S}$ & 0.6 \\
& Plant-gall & $\mathrm{SA}$ & 0.78 & $\mathrm{SA}$ & 0.45 \\
& Plant-mine & $\mathrm{E}$ & 0.73 & $\mathrm{SA}$ & 0.48 \\
& Plant-sca insect & $\mathrm{DS}$ & 0.73 & $\mathrm{D}$ & 0.51 \\
& Plant-aphid & $\mathrm{DA}$ & 0.65 & $\mathrm{D}$ & 0.53 \\
& Herbivore-parasitoid & $\mathrm{D}$ & 0.67 & $\mathrm{D}$ & 0.46
\end{tabular}

'This article is protected by copyright. All rights reserved.' 